\title{
ON THE MARKET
}

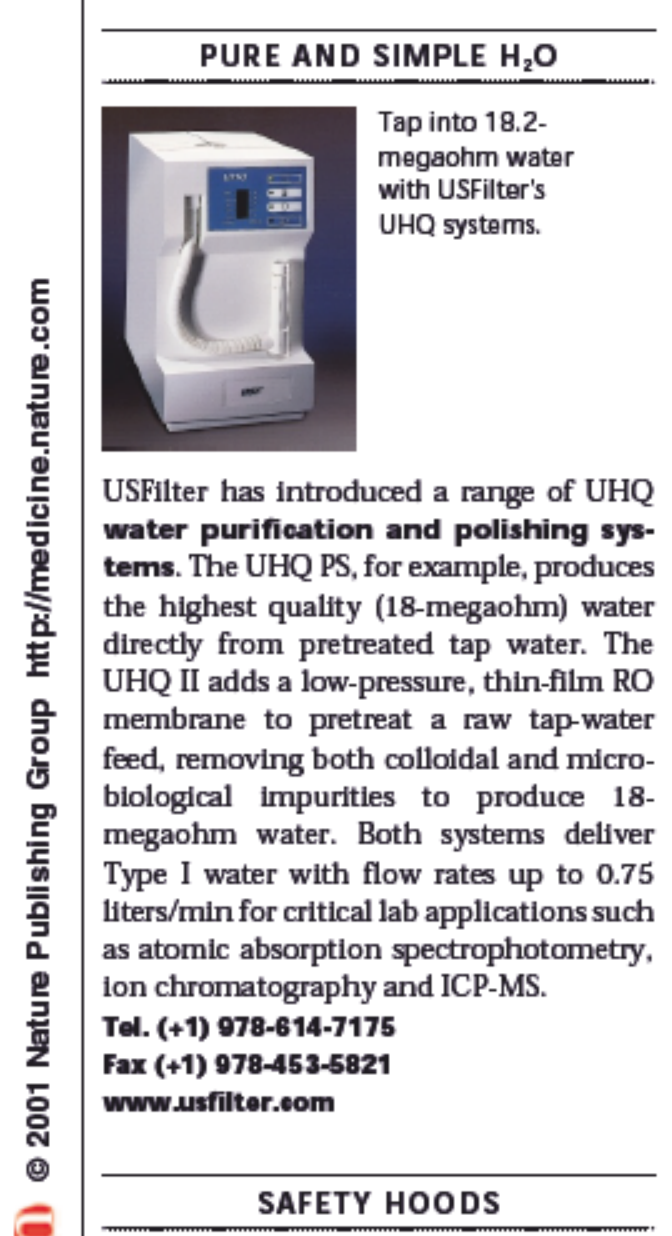

Astec Microflow has launched a new biological safety cabinet - the Microsafe Advanced Bio Safety Class II Cabinet. The cabinet features an ergonomic design with sloping front for ease of use and to provide a comfortable working position for operators. It recirculates approximately 70 percent of the air back into the working area. This air passes through a high-efficiency particulate air (HEPA) filter, as does the exhaust air, and the flows are continuously monitored by the cabinet. Any changes in air flow are automatically corrected by the microprocessorcontrolled fan system. This is also said to ensure that no part of the cabinet is under positive pressure to the laboratory - eliminating the risk of leaks. Other design features include a sliding. 'click-shut' door, which provides complete protection against fumigation, and safety alarms for air velocity and filter changes.

Tel. (+44) (0) 1934-418685

Fax (+44) (0) 1934-419033

www.astec-microflow.eo.uk

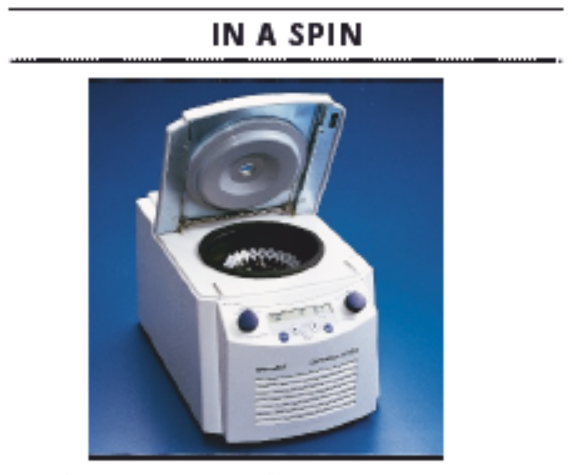

Refrigerated centrifuge cools samples quickly to between 0 and $40^{\circ} \mathrm{C}$.

The new refrigerated microcentrifuge from Eppendorf-Netheler-Hinz is a compact device that cools samples quickly in the temperature range of $0{ }^{\circ} \mathrm{C}$ and $40^{\circ} \mathrm{C}$ and reaches a top speed of 13,200 r.p.m. in $13 \mathrm{~s}$. Moreover, it can keep temperature-sensitive samples at $4{ }^{\circ} \mathrm{C}-$ even at maximum speed, says the company. The $5415 \mathrm{R}$ can accommodate a 24-position standard rotor for 1.5- and 2.0-ml tubes, or a 36-position rotor for $0.5-\mathrm{ml}$ tubes. Adapters for $0.2-\mathrm{ml}, 0.4-\mathrm{ml}, 0.5-\mathrm{ml}$ and 0.6-ml tubes are available for the standard rotor, with an additional adapter for 0.2-ml tubes provided for the special rotor. An aerosol-tight lid is also available for the standard rotor.

Tel. (+49) 40-53801-0

Fax (+49) 40-53801-556

wrww.eppendorf.com

The Sorvall Legend general purpose refrigerated tabletop centrifuge from Kendro Laboratory Products offers speeds to 15,000 r.p.m. $(21,890 \mathrm{~g})$ and a wide choice of biocontainment-approved rotors for applications that include tissue culture, virological testing and DNA or protein precipitation. Legend's rotors include a 4 x 750-ml swinging bucket rotor and the autoclavable BIOshield windshielded rotor for fast, high-capacity sample processing. The former accepts up to 16 standard microplates. The SmartSpin imbalance detection system provides optimum run stability and allows samples to be balanced by eye. Automatic rotor recognition is another standard feature. Control functions include a four-program memory for standard runs, RCF programming, nine braking and acceleration profiles, and a 'pretemp' facility for fast precooling.

Tel. (+1) 203-270-2203 (US)

Tel. (+49) 6181-35-5846 (Europo) $\frac{\text { MONOCLONALS }}{\text { Dako now offers all three classes of }}$ monoclonal antibodies to CD34, hematopoletic progenitor cell. The clone QBEnd 10 (class II) is available purified and FITC-conjugated. CD34 is expressed on stem and hematopoletic progenitor cells in bone marrow, umbilical cord blood and peripheral blood. It is also important for immunophenotyping of CD10+ ALL and AML cases. The company offers two FITC-conjugated CD34 antibodies. Each antibody represents a different class of epitope as defined by the sensitivity to degradation by the enzymes glycoprotease, neuramidase and chymopapain.

\section{Tel. (+1) 805-566-6655}

Fax (+1) 805-566-6688

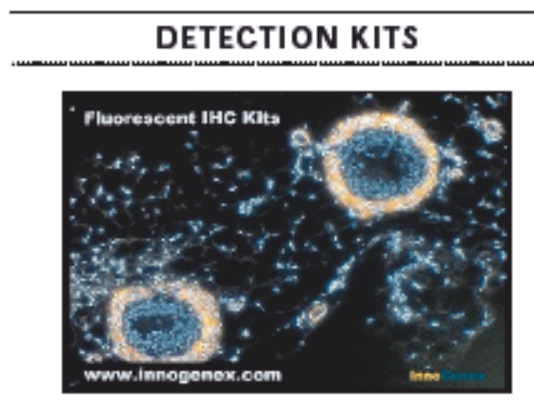

Ready-to-use kits for immunofluorescent staining of cells and tissues.

New ready-to-use immunofluoresoent kits from InnoGenex offer sensitive fluorescent detection of antibodies for immunohistochemical staining of cells and tissues. The technique uses enhanced biotin-streptavidin detection technology that provides high-resolution fluorescence staining of paraffin sections, cryostat sections, cell monolayers and whole mounts. A choice of four streptavidin-conjugated fluorescent dyes is available: Phycoerythrin (absorption 475-575 nm, emission peak $580 \mathrm{~nm}$ ), FluorBlue (abs. $350 \mathrm{~nm}$, emiss. $442 \mathrm{~nm}$ ), FluorGreen (abs. $488 \mathrm{~nm}$, emiss. $524 \mathrm{~nm}$ ) and FluorRed (abs. 519 $\mathrm{nm}$, emiss. 617-630 nm). A selection of biotinylated secondary antibodies is also offered, permitting the use of mouse, rabbit, rat or goat primary antbodies.

Tel. (+1) 925-543-1400

Fax (+1) 925-543-1405

www.innogenex.eom 